EPJ Web of Conferences 64, 05008 (2014)

DOI: $10.1051 /$ epjconf/ 20146405008

(C) Owned by the authors, published by EDP Sciences, 2014

\title{
Boundary Between Stable and Unstable Regimes of Accretion
}

\author{
A. A. Blinova ${ }^{1}$, a , R. V. E. Lovelace ${ }^{1}$, M. M. Romanova ${ }^{1}$ \\ ${ }^{1}$ Department of Astronomy, Cornell University, Ithaca, NY 14853-6801, USA
}

\begin{abstract}
We investigated the boundary between stable and unstable regimes of accretion and its dependence on different parameters. Simulations were performed using a "cubed sphere" code with high grid resolution ( 244 grid points in the azimuthal direction), which is twice as high as that used in our earlier studies. We chose a very low viscosity value, with alpha-parameter $\alpha=0.02$. We observed from the simulations that the boundary strongly depends on the ratio between magnetospheric radius $r_{m}$ (where the magnetic stress in the magnetosphere matches the matter stress in the disk) and corotation radius $r_{\text {cor }}$ (where the Keplerian velocity in the disk is equal to the angular velocity of the star). For a small misalignment angle of the dipole field, $\Theta=5^{\circ}$, accretion is unstable if $r_{\text {cor }} / r_{m}>1.35$, and is stable otherwise. In cases of a larger misalignment angle of the dipole, $\Theta=20^{\circ}$, instability occurs at slightly larger values, $r_{\text {cor }} / r_{m}>1.41$.
\end{abstract}

\section{Introduction}

Magnetospheric accretion occurs in different types of stars, including Classical T Tauri stars (CTTSs) [1], magnetized cataclysmic variables [2], and accreting millisecond pulsars [3]. Matter can accrete to magnetized stars in the stable regime, where ordered funnel streams are formed and subsequently flow to the magnetic poles, or in the unstable regime (see Fig. 1), where matter penetrates through the magnetosphere in several unstable tongues due to the magnetic Rayleigh-Taylor instability [4-6].

Stars accreting in stable and unstable regimes have different observational properties. In the stable regime, matter of the funnel streams falls to the star and forms two antipodal hot spots on its surface, and the expected light-curve is nearly sinusoidal. In the unstable regime, several hot spots form per rotational period of the inner disk, and the light-curve may look stochastic, with several peaks per period of rotation $[4,6]$. Such stochastic light-curves are also expected to be observed in the spectral lines, particularly in their red-shifted components [7]. Therefore, it is important to know which stars are expected to be in the stable or unstable regimes of accretion, and to derive the boundaries between these two regimes.

The main research objective was to investigate the boundary between stable and unstable regimes of accretion in simulations with a high grid resolution and at a low viscosity parameter, $\alpha=0.02$, in the disk.

\section{Method}

To investigate the boundary between stable and unstable regimes of accretion, we performed multiple 3D MHD (magnetohydrodynamic) simulation runs using a Godunov-type numerical code, written in

\footnotetext{
ae-mail: alisablinova@gmail.com
} 


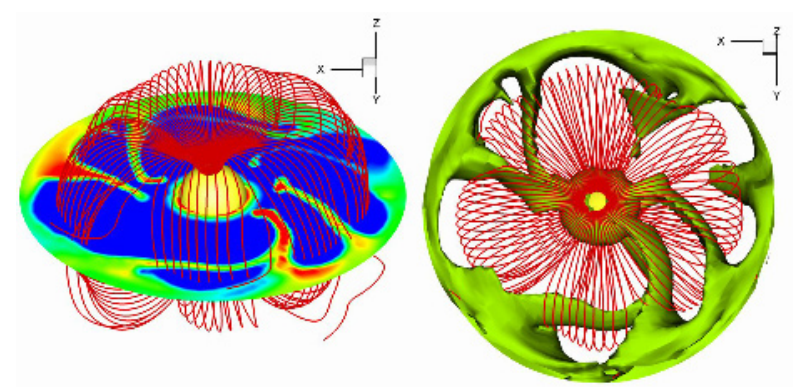

Figure 1. 3D views of accretion through Rayleigh-Taylor instability. Left panel: sample field lines and equatorial density distribution. Right panel: a 3D top-down view.

a "cubed sphere" coordinate system rotating with the star [8]. Simulations were performed using a high grid resolution (244 grid points in the azimuthal direction), which is twice as high as that used in our earlier studies. We considered accretion from an $\alpha$-disk [9] with a small $\alpha$-parameter, $\alpha=0.02$.

\section{Results}

In our simulations we varied the dipole magnetic moment $(\mu)$ from $\mu=0.05-3$, and the corotation radius from $r_{\text {cor }}=1.2-3$ (in dimensionless units). We found that the boundary strongly depends on the ratio of corotation radius $r_{\text {cor }}$ to magnetospheric radius $r_{m} . r_{m}$ was obtained from the simulations. We ran simulations for two different misalignment angles of the dipole, $\Theta=5^{\circ}$ and $\Theta=20^{\circ}$.

For a small misalignment angle of the dipole, $\Theta=5^{\circ}$, accretion is unstable if $r_{\text {cor }} / r_{m}>1.35$, and is stable otherwise (see Fig. 2, left panel). That is, instability occurs more easily for larger corotation radii and smaller magnetospheric radii. Alternatively, when $r_{m}$ approaches $r_{\text {cor }}$, accretion becomes stable. For example, when we take two cases with the same magnetic moment, $\mu=2$, but two different corotation radii, $r_{\text {cor }}=2$ and $r_{\text {cor }}=3$, we find that for the same moment in time the former case is stable, while the latter case is unstable (see Fig. 3). For a larger misalignment angle, $\Theta=20^{\circ}$, instability occurs at slightly larger values, $r_{\text {cor }} / r_{m}>1.41$ (see Fig. 2, right panel). This is probably due to the fact that at larger misalignment angles the magnetic poles are closer to the accretion disk plane, which makes funnel stream formation more favorable than the Rayleigh-Taylor instability.
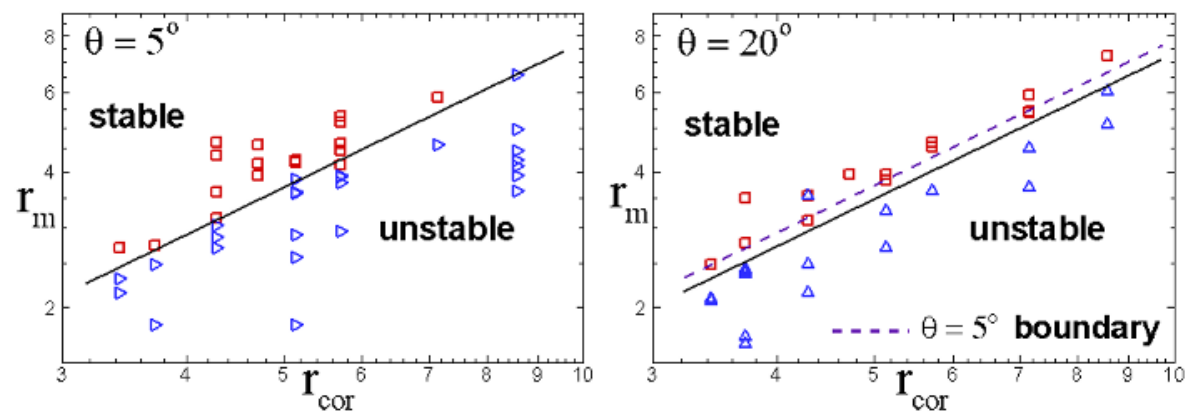

Figure 2. The boundary between stable and unstable regimes of accretion in the parameter space of $r_{m}$ and $r_{\text {cor }}$ for two different misalignment angles of the dipole. The units are given in stellar radii. Left panel: the boundary line for $\Theta=5^{\circ}$. Right panel: superposition of the $\Theta=5^{\circ}$ boundary line with the $\Theta=20^{\circ}$ data set and line. 

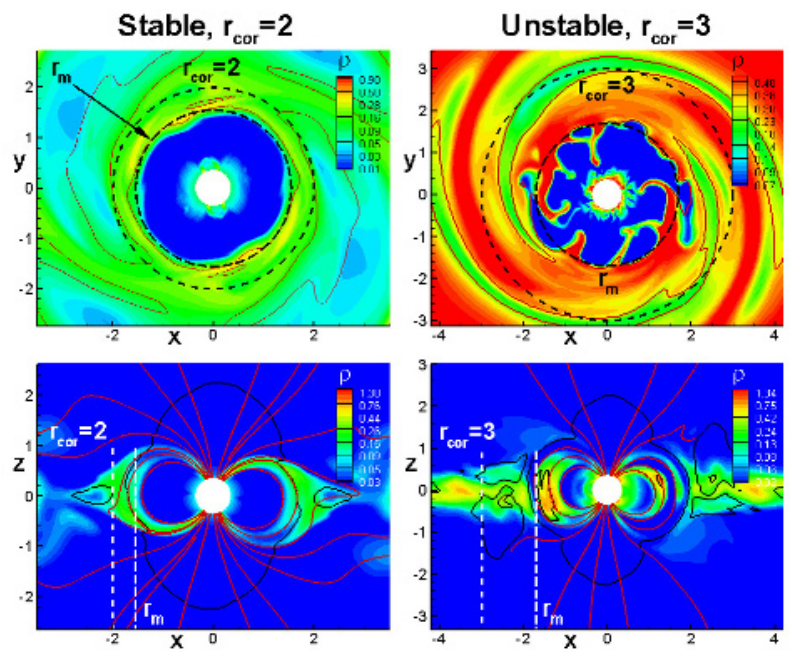

Figure 3. Examples of slices of density distribution in stable and unstable cases for $\Theta=5^{\circ}$, where dipole moment $\mu=2$ and only $r_{\text {cor }}$ is varied. Left panels: xy-slice (top) and xz-slice (bottom) show the case of $r_{\text {cor }}=2$, where accretion is stable. Right panels: accretion becomes unstable when $r_{\text {cor }}=3$. Here, the units are dimensionless, with $r_{\text {star }}=0.35$.

It should also be noted that not all cases were completely stable or completely unstable during a single simulation run. We broke these "intermediate" cases up into several stable and unstable parts at different moments in time, and recorded their corresponding magnetospheric radii.

We compared our simulation results with the analytical criterion for the existence of instability by Spruit et al. [10]:

$$
\gamma_{B \Sigma}^{2} \equiv g_{\mathrm{eff}}\left|\frac{d}{d r} \ln \frac{\Sigma}{B_{z}}\right|>2\left(r \frac{d \Omega}{d r}\right)^{2} \equiv \gamma_{\Omega}^{2}
$$

where $\Sigma$ is the surface density in the disk; $B_{z}$ is the z-component of the magnetic field; $\Omega$ is the angular velocity of the disk; $g_{\text {eff }} \equiv g-\Omega^{2} r$ is the effective gravitational acceleration $\left(g_{\text {eff }}\right.$ is positive if the acceleration is radially inwards). In other words, the $\Sigma / B_{z}$ and $g_{\text {eff }}$ terms should increase fast enough with $r$ to overcome the effects of the shear term of angular velocity, $\gamma_{\Omega}^{2}$, which has a stabilizing effect on the accretion disk. The majority of our cases for both misalignment angles are consistent with the Spruit criterion. That is, at the magnetospheric radius (where the unstable perturbations occur), $\gamma_{B \Sigma}^{2}>\gamma_{\Omega}^{2}$ for the unstable cases, while $\gamma_{B \Sigma}^{2}<\gamma_{\Omega}^{2}$ for the stable cases, which is consistent with the analytical prediction. See Fig. 4 for an example of the Spruit criterion applied to two cases with the same dipole magnetic moment, $\mu=2$, but different corotation radii.

Note that these simulations are different from those of $[4,6]$ in that we used $\alpha=0.02$ in all simulation runs (which approximately corresponds to constant accretion rate), while in $[4,6]$ the $\alpha$ parameter (and hence the accretion rate) varied. The comparison of our results with previous works shows that instability occurs more readily when using a finer grid. 


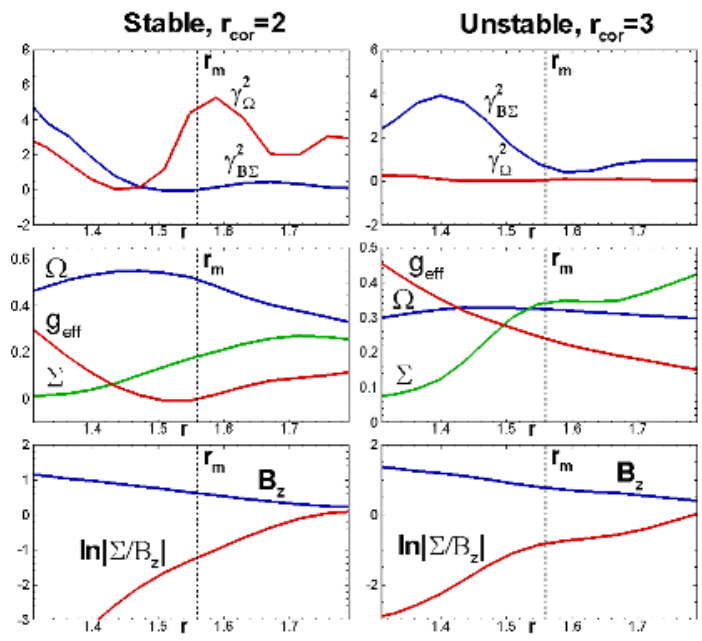

Figure 4. Radial distribution of different parameters in the disk and terms of the Spruit criterion in the vicinity of $r_{m}$. Dipole magnetic moment $\mu=2$ (in dimensionless units). $\Theta=5^{\circ}$. Accretion is stable for the smaller $r_{\text {cor }}$, $\gamma_{B \Sigma}^{2}<\gamma_{\Omega}^{2}$ (left panel), and unstable for the larger $r_{\text {cor }}, \gamma_{B \Sigma}^{2}>\gamma_{\Omega}^{2}$ (right panel).

\section{Summary}

The boundary between stable and unstable regimes of accretion strongly depends on the ratio $r_{\text {cor }} / r_{m}$. Accretion is slightly more stable for larger misalignment angles $\Theta$, with $r_{\text {cor }} / r_{m}$ having a slightly higher value. The results of our simulations are also consistent with the Spruit criterion for instability.

\section{Acknowledgments}

We thank the conference organizers, particularly Dr. E. Bozzo, for organizing an excellent conference. Resources supporting this work were provided by the NASA High-End Computing (HEC) Program through the NASA Advanced Supercomputing (NAS) Division at Ames Research Center and the NASA Center for Computational Sciences (NCCS) at Goddard Space Flight Center. The research was supported by NASA grant NNX11AF33G and NSF grant AST-1008636.

\section{References}

[1] Bouvier J., Alencar S. H. P., Harries T. J., Johns-Krull C. M., Romanova M. M., Protostars and Planets V, Eds. Reipurth B., Jewitt D., Keil K. (University of Arizona Press, Tucson, 2007) 479

[2] Warner B., PASP 116, 115 (2004)

[3] van der Klis M., Compact Stellar X-Ray Sources, Eds. Lewin W. H. G. and van der Klis M. (Cambridge Univ. Press, Cambridge, 2006) 39

[4] Kulkarni A. K. and Romanova M. M., MNRAS 386, 673 (2008)

[5] Arons J. and Lea S. M., ApJ 207, 914 (1976)

[6] Romanova M. M., Kulkarni A. K., Lovelace R. V. E., ApJ 673, L171 (2008)

[7] Kurosawa R. and Romanova M. M., MNRAS in press, e-print arXiv:1307.3639 (2013)

[8] Koldoba A. V., Romanova M. M., Ustyugova G. V., Lovelace R. V. E., ApJ 576, L53 (2002)

[9] Shakura N. I. and Sunyaev R. A., A\&A 24, 337 (1973)

[10] Spruit H. C., Stehle R., Papaloizou J. C. B., MNRAS 275, 1223 (1995) 